\title{
MASSIVE CONTACT BINARY SYSTEMS
}

\author{
Kam-Ching Leung \\ Behlen observatory \\ University of Nebraska \\ Lincoln, NE 68588-0111 \\ U.S.A.
}

\begin{abstract}
In recent years very massive single stars have keen found to be upward of $90 \mathrm{Mo}$. Massive contact binary systems have been found among the early-type systems, but their masses are far less than those reported for single :tars. The most massive component found is about 60 Mo.

It is generally believed that no late-type very massive stars have been detected (Humphreys and Davidson). This may be due to the large amount of mass loss from stellar wind. Recently, several extremely long-period late-type binary systems have been found to be contact systems. Two systems, IIJ Cnc and 5 cet, have their primary components with masses exceeding $40 \mathrm{Mo}$, and $K$ spectra. This result tends to suggest that close or interacting binary stars may be able to preserve the mass loss from stellar wind within the binary systems.
\end{abstract}

\section{INTRODUCTION}

Historically, contact binary systems were synonymous with $W$ UMa systems. W UMa contact binaries were normally associated with short-period, late spectral-type (G or later), and low mass (one solar mass or smaller) systems. A decade and a hialf ago contact systems consisting of massive components were discovered. At present, we have found contact systems in al.l spectral-types except for $M$ stars. ( This exeception could be due to the selection effect being too faint to be discovered effectively. There are only two confirmed ecilipsing systems with $M$ spectra and they are detached pairs.) Since we are primarily dealing with Wolf-Rayet stars and massive stars in this symposium, we will direct our attention to Wolf-Rayet, and massive type systems in this piaper. 


\section{MASSIVE EARLY-TYPE SYSTEMS}

If we make an arbitrary definition of massive stars being about 20 Mo or more, then there are 10 early-type contact systems in the class: 1 Wolf-Rayet systems, 70 type systems and 2 B type systems. Some of the spectra of these systems are complicated and the measurement of radial velocities are quite aifficult. About half of them have asymnetric light curves. The basic observed and derived quantities of these systems are summarized in Table 1 . There is still significant uncertainty concerning the spectral-type and tempera. ture relation among the very early-type stars. Thus, jt may be uriwise to employ the conventioral $H-R$ diagram, effective lemperature vs luminosity, since both axes involve temperature calibration. It may be more reliable to use a mass vs radius diagram. A $\log M / M_{0}$ vs $\log R / R o$ plot of these systems is shown in Figure 1. A straight line links up the two :omponents of the same system for identification. Notice that there is only one point for V348 Car in the diagram since the mass ratio of this system is unity. Six of the systems are found in the vicinity of the ZAMS and TAMS. This suggests that they were evolved contact sy:stems under Case A mass loss. On the other hard, V367 CYg, RY Sct, UW CMa, and V729 Cyg are found to be located above the TAMS line. It is suggested that these systems are evolved contact systems muler Case $B$ mass loss. These systems should be located $r e l a t i v e l y$ far away from the main sequence in a regular $H-R$ ij agram.

It is believed that very massive stars lose significant portions of their mass through stellar wind. The observations suggest that the very massive systems still evolve to the right of the main sequence, and go through the contact phase of binary evolution similar to the lower mass systems. The system with the latest spectra]-type is V367 Cyg (a member of $W$ ser star) with a B8 spectra. The mass, 19 Mo (< $40 \mathrm{MO})$, and the spectral-type, B8, of this system do not violate the Humphreys and Davidson (1979) limit.

\section{LATE-TYPE SUPERGIANT CONTACT SYSTEMS}

Recently, several late-type (G and $K$ ) hinary stars (5 Cet, UIT Cric, PW Pup, and possibly HDl04901B), with periods of a humbred days or longer, were found to be contact or near contact systems (Teung 1988). The shape of their lightcurves is very simjlar to those of $W$ UMa and $B$ Lyrae systems, except for their extremely long periodicity. Radial velocity curves are available for two of the systems (IJU cnc and 5 Cet). Both of them are found to be single-line spectroscopic: binary systems. Their absolute dimensions are determined by mearis of photometxic: mass ratios and the combiried photometric and spectroscopic solutions. The dimensions suggests 


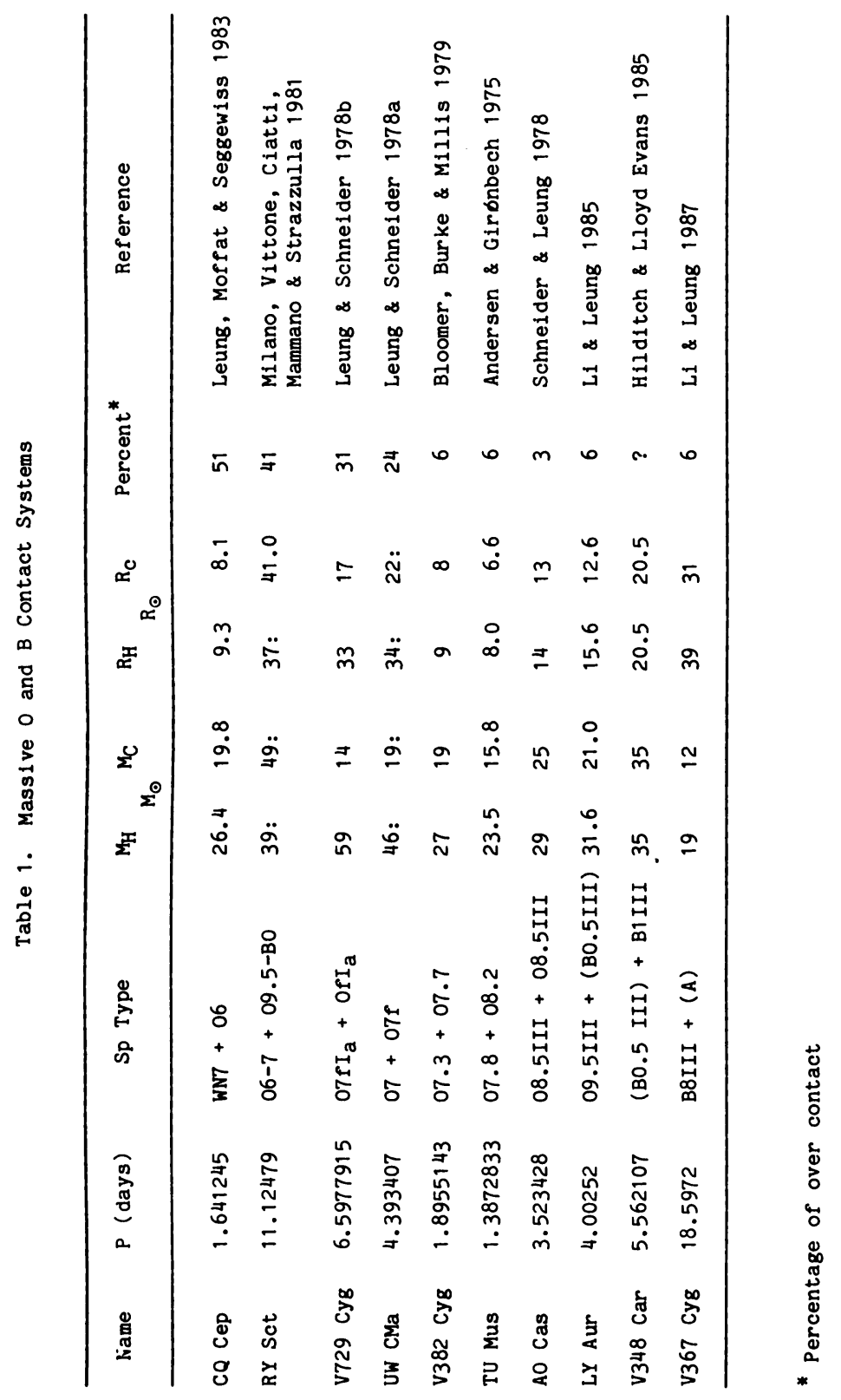




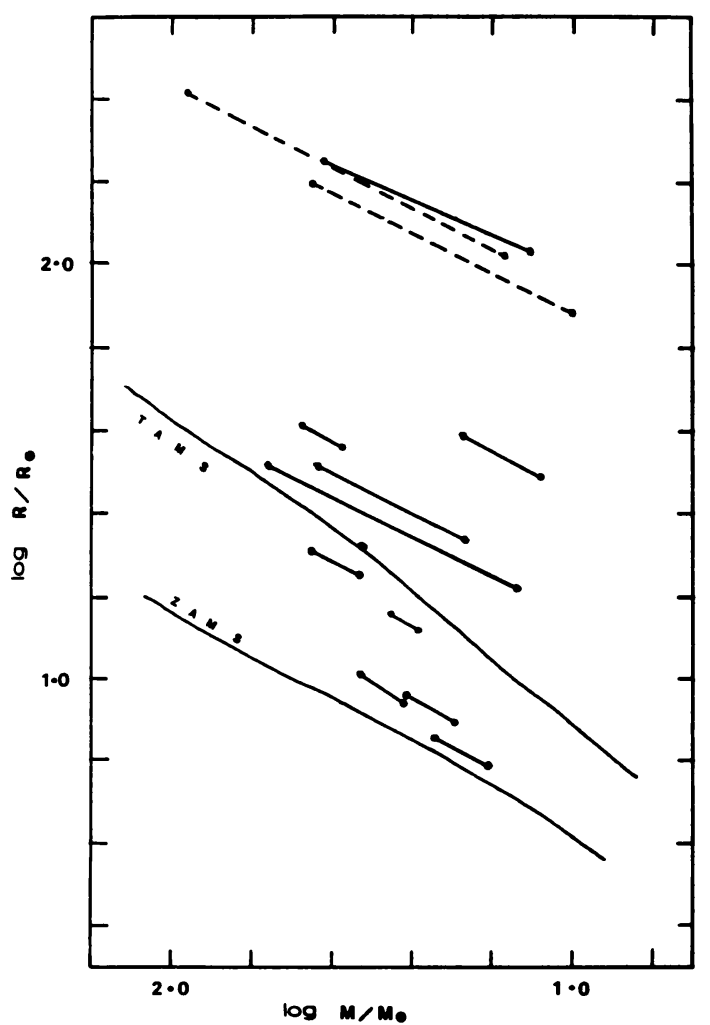

Figure 1. Log $M$ vs Log $R$ diagran of massive contact systens. Broken lines represent the two solutions of 5 Cet.

Table 2. Massive Late-Type Contact Systems

\begin{tabular}{|c|c|c|c|c|c|c|c|}
\hline Name & $P$ (days) & Sp Type & $M_{H}$ & $M_{\odot}{ }^{M_{C}}$ & $\mathrm{R}_{\mathrm{H}}$ & $\odot^{\mathrm{R}_{\mathrm{c}}}$ & Reference \\
\hline HD104901B & 106 & FO Ib-II & & & & & Leung 1988 \\
\hline PW Pup & 156 & F'epIab & & & & & Leung 1988 \\
\hline UU Cne & 96.7 & $\mathrm{~K} 2$ (4) & 44 & 13 & 184 & 109 & Lee, Nha \& Leung 1991 \\
\hline 5 Cet $^{*}$ & 96.4 & K2 & $\begin{array}{l}46 \\
94\end{array}$ & $\begin{array}{l}10 \\
15\end{array}$ & $\begin{array}{l}158 \\
264\end{array}$ & $\begin{array}{r}76 \\
108\end{array}$ & Li, Leung \& Ding 1988 \\
\hline
\end{tabular}

* See text 
that they are massive supergiant and giant stars. The basic okserved and derived quantities are listed in Table 2 . Note that there are two entries for 5 cet since there are two Vallues of photometric mass ratios, 0.21 and 0.16 from the photometric solutions. UU $\mathrm{Cnc}$ and 5 cet are located very far above the TAMS line. This suggests that these systems are evolved contact systems under advanced (very long after the hydrogen exhaustion phase of single star evolution) Case $B$ mass loss. These are very massive systems (both of the primary components are larger than $40 \mathrm{MO}$ ) and consist of late-type (K) supergiants. Generally, single stars (or noninteracting stars) follow the Humphreys and Davidson limit very well. However, for massive stars in an interacting sysitem to be able to evolve to the right of an H-R diagram, the system must be able to preserve mass loss from stellar wind. It would be very interesting to investigate the common envelopes of these systems. It is logical to interpret that the common envelope of such a system must corisist of a very dep convective atmosphere. It will be a real challenge to try to make theoretical models for this type of common atimosphere.

The author wishes to acknowledge the partial support of this research through grant INT-8616452 from the NSF.

\section{REFERENCES}

Aderser, J. and Gronbech, B. 1975, Astron. Astrophys., 45, 107.

Blcomex, R.H., Burk, E.W., King, C. and Millis, R.I.. 1979, Bul1. Amer. Astron. Soc., 11, 439.

Hilditch, R.W. and Lloyd Evars, T. 1985, Mor. Not. Roy. Astron. Soc., 213, 75 .

Humphreys, R.M. and Davidson K. 1979, Astrophys. J., 232, 409.

Jee, Y.S., Nha, I.S. and Jeung, K.C. 1991, Astrophys. J.'

Jeung, K.C. 1988, Critical Observations Vs Physical Models For Close Binary Stars ed. by K.C. Leung (New York: Gordon and Breach), p. 93.

Leing, K.C., Moffat, A.F.J. and Seggewis, W. 1983, Astrophys. J., 265, 961 .

Leung, K.C. and Schneider, D.P. 1978a, Astrophys. J., 222, 924 .

I.eurig, K.C. and Schreider, D.P. 1978b, Astrophys. J., 224, 565.

I.i, Y.F. and Leung, K.C. 1985, Astrophys. J., $298,345$.

I, i, Y.F. and Iseung, K.C. 1987, Astrophys. J., 313, 801.

Lj. Z.Y., Leung, K.C. and Ding, Y.R. 1988, Acta Astron. Sinica, 29,374 .

Milano, I., Vittone, A., Ciatti, F., Mammano, R. and Strazzulla, G. 1981, Astron. Astrophys, 100,59 .

Popper, D.M. 1980, Ann. Rev. Astron. Astropys., 18, 115.

Si:hneider, D.P. and Leung, K.C. 1978, Astrophys. J., 223, 202 . 


\section{DISCUSSION}

Niemela: Are there other determinations of masses for the red stars that you mentioned, and how do those values compare with yours?

Leung: Both systems are simple line spectroscopic binaries. For 5 Cet, Eaton estimated a mass ratio from line profile and obtained small masses. For UU Cnc, there were estimates from photometric analysis. Unfortunately, they only searched for ratios near unity. That is, they found solutions in localized minima in the $\bar{Z}-g$ plane. The global minimum is around 0.3 instead of near unity!

Vanbeveren: I have a comment on the influence of radiation pressure on the evolution and more specifically on the equipotential surfaces in close binaries. Actually from a evolutionary point of view you are interested in what is going on during the Roche lobe overflow process. Now this process can be considered as two processes: you first have the existence of a critical surface, from where you have very huge mass loss to some kind of Lagrangian point and than you have a second process which has left the star. And how does this behave in such a binary? Now I have shown that if you account for shadow effects then the radiation pressure does not modify at all the equipotential surface. E.g., if you assume the Von Zipal theorem, then you simply come up with the same equipotential surfaces as the usual of Roche lobe. But if you have two massive stars in a binary, once the matter has left the star as a consequence of this critical equipotential surface than you may not use any more, according to me at least, the classical computation done for two stars considered as two point masses, as has been done for low mass binaries in the massive binaries. Because there, in the computation of the trajectories of the particles, may be forming an accretion disk, you have to include radiation pressure, as has been done in the stellar wind theory. And if you do that, then the particles may acquire a velocity which is much larger than the escape velocity of the binary. And this was actually the main concern of the influence of radiation pressure on the Roche lobe. It has to be considered as the influence of radiation pressure on the Roche lobe overflow proces as a whole.

Kam-Ching Leung

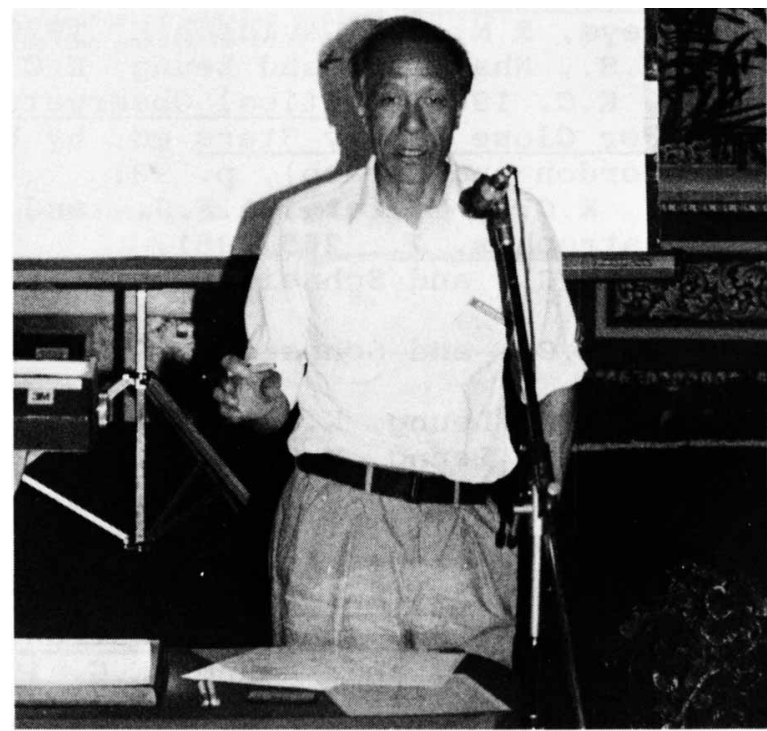

